В.3. Геллер, М.О. Шимчук, С.М. Губанов

Одеська національна академія харчових технологій, вул. Канатна 112, Одеса, 65039, Україна

\title{
ВЛАСТИВОСТІ ПЕРЕНОСУ НАНОФЛЮЇДІВ (ЕКСПЕРИМЕНТ I МЕТОДИ РОЗРАХУНКУ)
}

У статті наведено огляд та аналіз експериментальних досліджень $і$ методів розрахунку теплопровідності $i$ в'язкості нанофлюідів $з$ частинками $\mathrm{Al}_{2} \mathrm{O}_{3}$. Розглянуто вплив основних факторів на властивості переносу нанофлюідів, включаючи концентрацію, розмір $i$ форму наночастинок, спосіб отримання нанофлюідів $i$ т.д. Наведено результати експериментального дослідження теплопровідності і в'язкості модельної системи ізопропіловий спирт - наночастинки $\mathrm{Al}_{2} \mathrm{O}_{3}$ при різних конщентраціях наночастинок $i$ температурах. Дослідження теплопровідності проводилося двома незалежними методами - стаціонарним $i$ нестаціонарним методами нагрітої нитки. В'язкість вимірювалася за допомогою капілярних віскозиметрів. Досліджено також вплив добавки наночастинок на в'язкість холодильного компресорного масла. Результати вимірювань представлені у вигляді простих моделей.

Ключові слова: Теплопровідність - В'язкість - Нанофлюіди - Ізопропіловий спирт $\mathrm{Al}_{2} \mathrm{O}_{3}-$ Компресорне мастило

\section{В.3. Геллер, Н.А. Шимчук, С.Н. Губанов}

Одесская национальная академия пищевых технологий, ул. Канатная 112, Одесса, 65039, Украина

\section{СВОЙСТВА ПЕРЕНОСА НАНОФЛЮИДОВ (ЭКСПЕРИМЕНТ И МЕТОДЫ РАСЧЁТА)}

\begin{abstract}
В статье приведен обзор и анализ экспериментальных исследований и методов расчёта теплопроводности и вязкости нанофлюидов с частицами $\mathrm{Al}_{2} \mathrm{O}_{3}$. Рассмотрено влияние основных факторов на свойства переноса нанофлюидов, включая концентрацию, размер и форму наночастии, способ получения нанофлюидов и т.д. Приведены результаты экспериментального исследования теплопроводности и вязкости модельной системы изопропиловый спирт - наночастицы $\mathrm{Al}_{2} \mathrm{O}_{3}$ при различных концентрациях наночастиц $u$ температурах. Исследование теплопроводности проводилось двумя независимыми методами - стационарными и нестациионарным методами нагретой нити. Вязкость измерялась с помощьью капиллярных вискозиметров. Исследовано также влияние добавки наночастии на вязкость холодильного компрессорного масла. Результаты измерений представлень в виде простьх моделей.

Ключевые слова: Теплопроводность - Вязкость - Нанофлюиды - Изопропиловый спирт $-\mathrm{Al}_{2} \mathrm{O}_{3}-$ Компрессорное масло
\end{abstract}

\section{І. ВВЕДЕНИЕ}

Результаты исследований свойств нанофлюидов, опубликованные в последние годы, показывают возможность существенного целенаправленного изменения их теплофизических и теплообменных характеристик по сравнению с традиционными рабочими телами и теплоносителями. В ряде работ получено значительное увеличение теплопроводности и рост коэффициентов теплоотдачи при различных режимах течения в каналах различной формы и при фазовых переходах. Для объяснения причин этого явления анализируется несколько основных механизмов: броуновское движение наночастиц, образование высокотеплопроводного жидкого слоя (с толщиной молекулярного уровня) на границе раздела жидкость-наночастица, баллистический перенос тепловой энергии внутри индивидуальной наночастицы и между наночастицами, который происходит при их контакте, a также влияние кластеризации наночастиц. Возможность улучшения энергетических характеристик холодильных систем за счёт этого эффекта в настоящее время изучается. Например, в ряде работ показано, что наночастицы могут быть использованы в качестве добавки для повышения растворимости минерального масла с HFC-хладонами, при этом улучшаются возврат масла в компрессор и энергетические характеристики холодильной системы (СОР увеличивается на 10-15\%).

Следует также отметить, что важной характеристикой для разработки и оптимизации 
технологических теплообменных аппаратов, в которых циркулирует поток теплоносителя, является вязкость, поскольку это свойство определяет затраты энергии на прокачку теплоносителей и значительно влияет на эффективность процессов теплообмена.

Вместе с тем, экспериментальные исследования теплопроводности и вязкости нанофлюидов, проведенные к настоящему времени, весьма ограничены и разноречивы, а проблемой методы расчёта этих свойств является отсутствие универсальности (они применимы только к конкретным приложениям) и физического смысла.

В связи с этим, целью настоящей работы явились краткий обзор и анализ экспериментальных данных и методов расчёта теплопроводности и вязкости нанофлюидов, а также экспериментальное исследование суспензий с наночастицами окиси алюминия $\left(\mathrm{Al}_{2} \mathrm{O}_{3}\right)$.

\section{II. ОБЗОР И АНАЛИЗ ЭКСПЕРИМЕНТАЛЬНЫХ ИССЛЕДОВАНИЙ}

В таблице 1 приведена информация об основных опубликованных исследований теплопроводности нанофлюидов с частицами $\mathrm{Al}_{2} \mathrm{O}_{3}$. В этих работах в качестве базовых жидкостей использованы вода и этиленгликоль (имеется ещё несколько публикаций, в которых качестве базовых жидкостей использованы моторное, компрессорное и трансформаторное масла и глицерин).

Таблица 1 - Исследования теплопроводности нанофлюидов с частицами $\mathrm{Al}_{2} \mathrm{O}_{3}$

\begin{tabular}{|c|c|c|c|}
\hline $\begin{array}{c}\text { Концентра } \\
\text { ция частиц, } \\
\%\end{array}$ & $\begin{array}{c}\text { Размер } \\
\text { частиц, нм }\end{array}$ & $\begin{array}{c}\text { Макс. } \\
\text { эффект } \\
\lambda_{\mathrm{H}} / \lambda_{\sigma}, \%\end{array}$ & $\begin{array}{c}\text { Метод } \\
\text { измерений }\end{array}$ \\
\hline \multicolumn{4}{|c|}{ Базовая жидкость - вода } \\
\hline 4 & 38 & 9 & МТК \\
\hline 4 & 131 & 24 & МПС \\
\hline 4,4 & 13 & 33 & $\mathrm{HMHH}$ \\
\hline 4,3 & 38 & 11 & $\mathrm{HMHH}$ \\
\hline 5,5 & 28 & 16 & $\mathrm{M \Pi C}$ \\
\hline $1-4$ & $11-150$ & 30 & $\mathrm{HMHH}$ \\
\hline 10 & $36-47$ & 29 & МПС \\
\hline 5 & 11 & 8 & НМНКН \\
\hline 5 & $11-40$ & 13 & $\mathrm{HMHH}$ \\
\hline 5 & 60 & 21 & НМНH \\
\hline 1 & 48 & 4 & HМHH \\
\hline 1 & 80 & 12 & $\mathrm{HMHH}$ \\
\hline 4 & 45 & 13 & $3-\omega$ \\
\hline \multicolumn{4}{|c|}{ Базовая жидкость - этиленгликоль } \\
\hline 5 & 38 & 19 & $\mathrm{HMHH}$ \\
\hline 8 & 28 & 41 & МПС \\
\hline 5 & 35 & - & HMHH \\
\hline 5 & $26-300$ & 30 & $\mathrm{HMHH}$ \\
\hline 3 & 20 & 14 & $\mathrm{HMHH}$ \\
\hline 0,5 & 150 & 10 & HМHH \\
\hline 4 & 45 & 10 & $3-\omega$ \\
\hline
\end{tabular}

*) МТК - метод температурных колебаний, МПС метод плоского слоя, НМНН - нестационарный метод нагретой нити, НМНКН - нестационарный

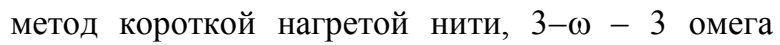
метод.

Анализ опубликованных данных показывает, что полученные в них результаты не согласуются между собой, при этом наибольшее влияние на приведенную теплопроводность нанофлюидов $\lambda_{H} / \lambda_{\sigma}$ (где $\lambda_{H}-$ теплопроводность нанофлюида, $\lambda_{\sigma}-$ теплопроводность базовой жидкости) оказывает концентрация наночастиц.

Кроме концентрации, из основных факторов, влияющих на теплопроводность нанофлюидов, наиболее важными являются следующие: способ получения нанофлюида и методика его подготовки к исследованиям; размер и форма исходных наночастиц; способность наночастиц образовывать кластеры, размеры кластеров; температура и давление; поверхностно-активные вещества (сурфактанты); тип и свойства базовых жидкостей; методика проведения эксперимента и её адаптация к специфике нанофлюидов, влияние конвективной составляющей и эффекта «печной трубы», влияние диффузионных эффектов, влияние излучения и переизлучения для нанофлюидов на основе базовых жидкостей, полупрозрачных в ИКобласти спектра и т.д. Анализ этих факторов приведен в нашей статье [1], в которой показано, что, например, по влиянию температуры результаты опубликованных работ прямо противоположны - часть из них показывает уменьшение эффекта влияния наночастиц на теплопроводность базовой жидкости с ростом температуры, другая часть - увеличение этого эффекта.

В таблице 2 представлена информация об основных опубликованных исследованиях вязкости нанофлюидов.

Таблица 2 - Опубликованные данных по вязкости нанофлюидов

\begin{tabular}{|c|c|c|c|c|}
\hline $\begin{array}{c}\text { Базовая } \\
\text { жидкость }\end{array}$ & $\begin{array}{c}\text { нип и размер } \\
\text { нм }\end{array}$ & $\begin{array}{c}\text { Конц. } \\
\text { наночас } \\
\text { тиц, \% }\end{array}$ & $\begin{array}{c}\text { Интервал } \\
\text { темп-ур, } \\
{ }^{\circ} \mathrm{C}\end{array}$ & $\begin{array}{c}\text { Макс. } \\
\text { эффект, } \\
\%\end{array}$ \\
\hline ЭГ & $\mathrm{TiO}_{2}(25)$ & $0,5 \div 8$ & $20 \div 60$ & 22 \\
\hline $\begin{array}{c}\text { Синт. } \\
\text { масло }\end{array}$ & $\mathrm{TiO}_{2}(21)$ & $0,0 \div 0,5$ & $10 \div 90$ & 9 \\
\hline Вода & $\mathrm{TiO}_{2}(76)$ & $1 \div 35$ & $10 \div 70$ & 240 \\
\hline Синт. & $\mathrm{CuO}(30)$ & $3 \div 39$ & $15 \div 45$ & 400 \\
масло & $\mathrm{CuO}(29)$ & $1 \div 6$ & $-35 \div 50$ & 400 \\
\hline ЭГ/Вода & $\mathrm{Al}_{2} \mathrm{O}_{3}(30-$ & $1 \div 13$ & $20 \div 60$ & 400 \\
\hline Вода & $80)$ & 13 & $20 \div 60$ & 433 \\
& $\begin{array}{c}\mathrm{TiO}_{2}(30-80) \\
\mathrm{SiO}_{2}(30-80)\end{array}$ & 31 & $20 \div 60$ & 400 \\
\hline Вода & $\begin{array}{c}\mathrm{Al}_{2} \mathrm{O}_{3}(30) \\
\mathrm{SiO}_{2}(12) \\
\mathrm{TiO}_{2}(21)\end{array}$ & 0,$5 ; 1,5$ & $20 \div 50$ & $75 \div 4$ \\
$0,2 \div 3$ & $20 \div 50$ & $42 \div 55$ & 130 \\
\hline Вода & $\mathrm{Al}_{2} \mathrm{O}_{3}(25)$ & $0,5 \div 5$ & $20 \div 50$ & 850 \\
\hline
\end{tabular}




\begin{tabular}{|c|c|c|c|c|}
\hline ЭГ & $\mathrm{Al}_{2} \mathrm{O}_{3}(25)$ & $0,5 \div 5$ & $20 \div 50$ & 220 \\
\hline ЭГ/Вода & $\mathrm{Al}_{2} \mathrm{O}_{3}(45)$ & $1 \div 10$ & $20 \div 90$ & 260 \\
\hline
\end{tabular}

*) ЭГ - этиленгликоль

Анализ показал, что вязкость зависит от температуры, концентрации, размера и типа наночастиц, а также от способа приготовления нанофлюидов, однако количественные оценки этих зависимостей из опубликованных данных получить не удалось. Например, представленные на рисунке 1 данные о приведенной динамической вязкости $\eta / \eta_{0}$ (где $\eta_{0}$ - вязкость базовой жидкости) для одних и тех же нанофлюидов с одинаковыми размерами наночастиц, полученные в работах [2] и [3], отличаются между собой в 4-5 раз.

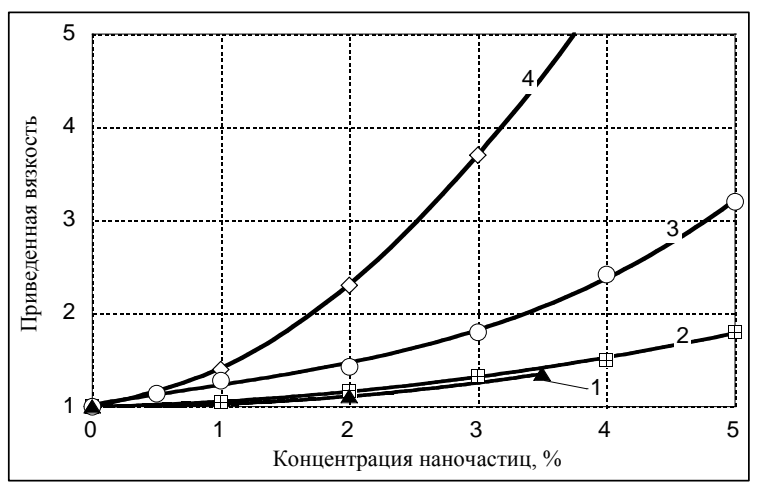

Рисунок 1 - Зависимость приведенной вязкости от объёмной концентрации наночастиц $1-\mathrm{Al}_{2} \mathrm{O}_{3}-E G(28 \mathrm{~nm})$ [3], $2-\mathrm{Al}_{2} \mathrm{O}_{3}-\operatorname{water}(28 \mathrm{~nm})$ [3], $3-\mathrm{Al}_{2} \mathrm{O}_{3}-E G(25 \mathrm{~nm})$ [2], $4-\mathrm{Al}_{2} \mathrm{O}_{3}$-water (25 $\mathrm{nm})$ [2]

Противоречивость экспериментальных данных обусловлена естественными трудностями проведения опытов: не удается создать монодисперсную наножидкость, практически не контролируется распределение частиц по размерам. Кроме того, с ростом концентрации частиц наножидкость может менять реологию.

\section{III. МЕТОДЫ РАСЧЁТА ТЕПЛОПРОВОДНОСТИ И ВЯЗКОСТИ НАНОФЛЮИДОВ}

В настоящее время нет надежной теории предсказания аномальной теплопроводности наножидкостей. Тем не менее, существует несколько полуэмпирических соотношений для расчета теплопроводности двухфазных смесей. Эти модели можно условно разделить на классические и динамические. Классические модели, в основном, базируются на работе Максвелла [4], в которой получено уравнение (1)

$$
k_{\text {eff }}=\frac{k_{p}+2 k_{b}+2\left(k_{p}-k_{b}\right) \phi}{k_{p}+2 k_{b}-\left(k_{p}-k_{b}\right) \phi} k_{b}
$$

для теплопроводности суспензий с относительно крупными сферическими частицами при их небольших концентрациях.

Частным случаем модели Максвелла является модель Гамильтона и Кроссера [5] для суспензий с несферическими частицами. Они ввели фактор формы $n$ для учета влияния формы наночастиц (2)

$$
k_{e f f}=\frac{k_{p}+(n-1) k_{b}-(n-1)\left(k_{b}-k_{p}\right) \phi}{k_{p}+(n-1) k_{b}+\left(k_{b}-k_{p}\right) \phi} k_{b} .
$$

Классические модели выводятся из предположения сплошной среды и включают только форму частиц и их объемную долю, при этом предполагается диффузный перенос тепла в обеих жидкой и твердой фазах. Развитие динамических моделей шло по пути учёта и других возможных эффектов, в том числе броуновского движения наночастиц, термического сопротивления межфазного слоя, теплопроводности самих наночастиц, кластеризации наночастиц и т.д.

Существующие методы расчёта вязкости нанофлюидов базируются, в основном, на модификациях модели Эйнштейна [6], в которой для вязкости нанофлюидов со сферическими твердыми частицами использованы феноменологические уравнения гидродинамики с учётом влияния частиц на гидродинамическое поле скорости несущей жидкости и, как следствие, на тензор напряжений и коэффициент вязкости (3)

$$
\eta_{n f}=\eta_{f}\left[1+(5 / 2) \varphi_{n}\right]
$$

где $\eta_{f}$ - вязкость базовой жидкости, $\varphi$ - объемная концентрация дисперсных частиц.

Это уравнение получено для бесконечно разбавленного раствора, содержащего сферические не взаимодействующие между собой частицы. Последующие эксперименты показали, что формула (3) справедлива лишь при $\varphi<10^{-3}$.

Принципиальным фактом является то, что теория Эйнштейна не описывает поведение эффективной вязкости наножидкостей даже при малых концентрациях наночастиц. При низких концентрациях наночастиц коэффициент вязкости слабо зависит от размера частиц. Однако, с ростом концентрации коэффициент вязкости наножидкостей $\mathrm{c}$ более мелкими частицами оказывается существенно выше, чем у наносуспензий с крупными частицами.

Позднее предпринимались многочисленные попытки обобщить теорию Эйнштейна на случай более высоких концентраций наночастиц. Эти работы можно подразделить на три группы. В первой из них гидродинамическими методами определялись поправки к полям скоростей и давлений базовой жидкости, обусловленные взаимовлиянием дисперсных частиц. Во второй группе работ эффективный коэффициент вязкости нанофлюидов определялся

методами 
неравновесной статистической механики. Наконец, к третьей группе относятся работы, в которых поля скоростей и давлений моделируются некоторыми стохастическими законами. Во всех перечисленных теориях получаются соотношения вида (4)

$$
\eta_{n f}=\eta_{f}\left(1+2,5 \varphi_{n}+k_{H} \varphi_{n}^{2}\right)
$$

Соотношения такого вида несколько расширить диапазон позволили описания экспериментальных данных о вязкости нанофлюидов, при этом лучшие результаты даёт уравнение Бетчелора [7], учитывающее как гидродинамический эффект, так и эффект, связанный с броуновским движением жестких сферических наночастиц (5)

$$
\eta_{n f}=\eta_{f}\left(1+2,5 \varphi_{n}+6,2 \varphi_{n}^{2}\right)
$$

Вместе с тем, эти модели не позволяют получить приемлемые результаты. Так расчёт вязкости для системы вода $/ \mathrm{Al}_{2} \mathrm{O}_{3}$ и эксперимент при объёмной концентрации наночастиц 5\% отличаются между собой в несколько раз.

\section{IV. ЭКСПЕРИМЕНТАЛЬНОЕ \\ ИССЛЕДОВАНИЕ ТЕПЛОПРОВОДНОСТИ И ВЯЗКОСТИ НАНОФЛЮИДОВ}

Объектом исследования была выбрана модельная система изопропиловый спирт наночастицы $\mathrm{Al}_{2} \mathrm{O}_{3}$. Исследование теплопроводности проводилось двумя независимыми методами - стационарным и нестационарным методами нагретой нити. Методика измерений теплопроводности и некоторые экспериментальные результаты приведены нами в работе [1]. Стационарный метод нагретой нити, как показал анализ, позволяет получать надёжные данные для жидкостей стабильного во времени состава. Однако, для нанофлюидов ключевой проблемой остается их устойчивость. Кластеризация способствует объединению наночастиц и их выпадению в осадок, что приводит к временному изменению концентрации наночастиц и динамике теплофизических свойств нанофлюидов. В связи с этим, большая часть измерений теплопроводности получена с помощью нестационарного метода нагретой нити, который обладает достаточной точностью и чрезвычайно экспрессивен (процесс измерения занимает не более нескольких секунд).

Вязкость измерялась с помощью капиллярных вискозиметров с «висячим» уровнем [8]. При выборе объектов исследования вязкости были сформулированы следующие основные задачи:

- исследовать вязкость модельной системы изопропиловый спирт (изопропанол)/наночастицы $\mathrm{Al}_{2} \mathrm{O}_{3}$ при различных концентрациях наночастиц и температурах для определения влияния этих факторов на эффективную вязкость;

- исследовать влияние добавки наночастиц на вязкость технически важной жидкости холодильного компрессорного масла.

Вторая задача связана с тем, что добавка наночастиц, как следует из опубликованных в последние годы работ, обеспечивает определённые преимущества нанофлюидов по сравнению с традиционными рабочими веществами холодильных машин.

Следует отметить, что добавка наночастиц в холодильные компрессорные масла существенно увеличивают их вязкость, однако присутствие сурфактанта (роль которого может играть изопропанол) обеспечивает противоположный эффект. Таким образом, Решение поставленных задач потребовало также измерения вязкости базовых жидкостей - изопропанола (ИС), холодильного компрессорного масла (XМ) и масло-изопропаноловых смесей.

Результаты измерений теплопроводности и вязкости представлены на рисунках 2 и 3.

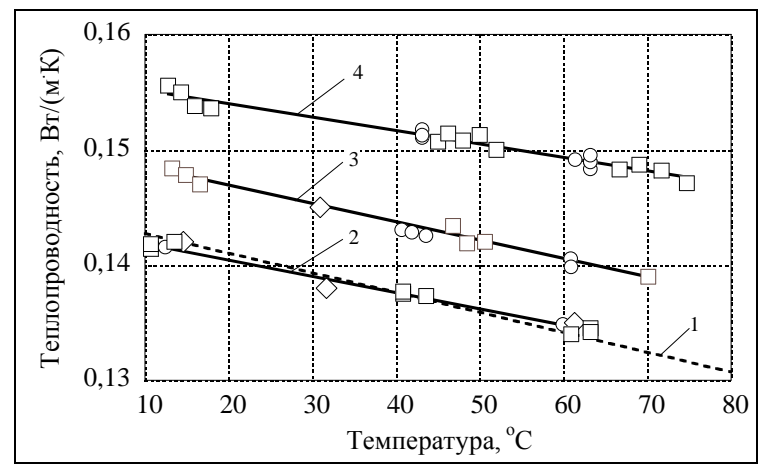

Рисунок 2 - Зависимость теплопроводности от температуры

1 - ИС (справочные данные), 2 - ИС, $3-И C+1,19 \% \mathrm{Al}_{2} \mathrm{O}_{3}, 4-И C+2,64 \%$

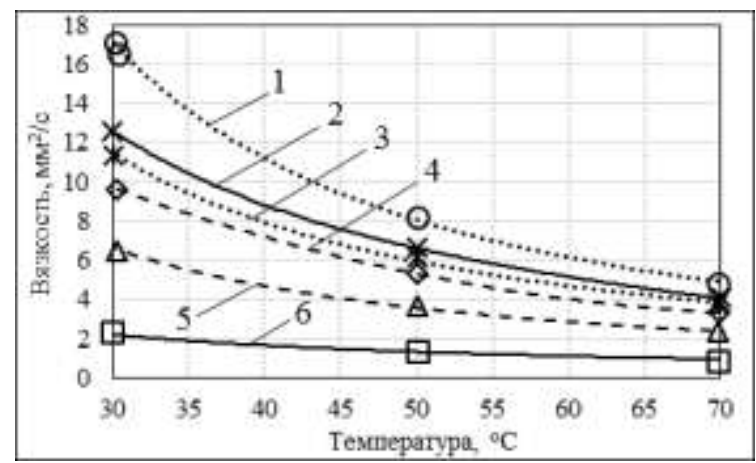

Рисунок 3 - Зависимость вязкости от температуры:

$1-X M / И C / \mathrm{Al}_{2} \mathrm{O}_{3}\left(x_{1}=8,8 \% ; x_{2}=2,2 \%\right) ; 2-X M ; 3-$ $X M / И C / \mathrm{Al}_{2} \mathrm{O}_{3}\left(x_{1}=9,4 \% ; x_{2}=1,1 \%\right)$;

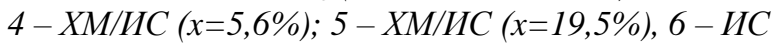

Анализ полученных данных показал, что добавка наночастиц существенно увеличивает вязкость жидкости, а присутствие сурфактанта (роль которого в наших опытах с холодильным 
компрессорным маслом играл маловязкий изопропанол) обеспечивает противоположный эффект. Результаты измерений вязкости компрессорного масла и смесей этого масла с изопропанолом и наночастицами $\mathrm{Al}_{2} \mathrm{O}_{3}$, представленные на рис. 3, иллюстрируют это заключение. Таким образом, подобрав соотношение концентраций наночастиц и сурфактанта можно «отрегулировать» стандартную вязкость и индекс вязкости холодильного компрессорного масла в необходимых пределах.

\section{V. АНАЛИЗ И ОБРАБОТКА РЕЗУЛЬТАТОВ ИЗМЕРЕНИЙ}

Экспериментальные данные системы изопропиловый спирт - $\mathrm{Al}_{2} \mathrm{O}_{3}$ были обработаны в виде зависимости приведенной теплопроводности $\lambda_{\mu} \lambda_{\sigma}$ от температуры и концентрации наночастиц (6)

$$
\lambda_{H} / \lambda_{\sigma}=1+\left(3,36 \cdot 10^{-2}+5,0 \cdot 10^{-4} \cdot t\right) \cdot x
$$

Отклонения всех результатов измерений, включая базовую жидкость и нанофлюиды, не превышают $1,5 \%$.

Проведено также сравнение результатов измерений теплопроводности $\mathrm{c}$ расчетом по классической модели Максвелла и еe модификациями Гамильтона и Кроссера для сферических и цилиндрических наночастиц. Это сравнения представлено на рисунке 4. Как видно из этого графика, лучшее совпадение экспериментальных данных имеет место при расчете по моделям Максвелла и Гамильтона Кроссера для цилиндрических наночастиц.

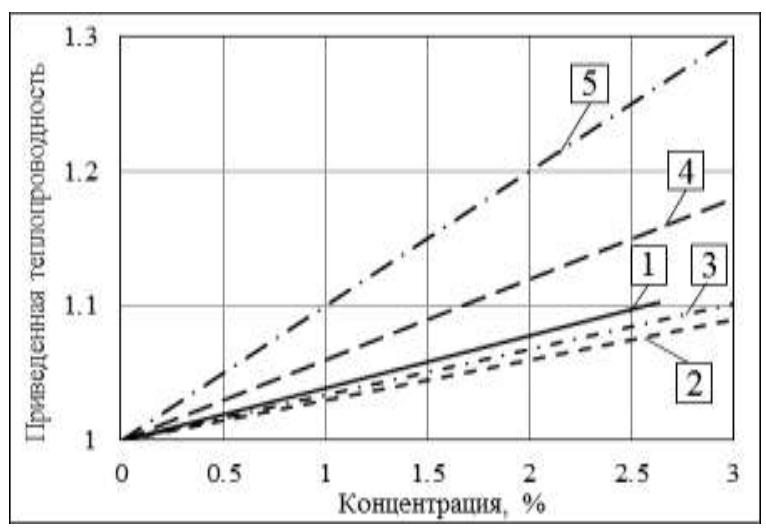

Рисунок 4 - Сравнение экспериментальных данных по теплопроводности с расчетом для различных моделей.

1 - эксперимент при $40{ }^{\circ} \mathrm{C}, 2$ и 3 -расчет по моделям Максвела и Гамильтона - Кроссера для ичилиндрических наночастии, 4 и 5 -расчет по модели Гамильтона - Кроссера для сферических наночастиц.

Анализ результатов измерений вязкости системы изопропиловый спирт - $\mathrm{Al}_{2} \mathrm{O}_{3}$ показал, что эффект влияния наночастиц на вязкость зависит от температуры. Возможно, с увеличением температуры и подвижности молекул кластеры наночастиц начинают распадаться, что и приводит к уменьшению эффекта влияния наночастиц на вязкость базовой жидкости. В связи с этим, экспериментальные данные были обработаны в виде зависимости приведенной вязкости $\eta / \eta_{0}$ от температуры и объёмной концентрации наночастиц $x_{\text {об }}(7)$

$$
\eta / \eta_{0}=1+\left(8,24 \cdot 10^{-1}-4,57 \cdot 10^{-3} \cdot t\right) \cdot x_{\text {об }}
$$

Сравнение экспериментальных данных и результатов расчета по уравнению (2) показало, что расхождения не превышают $3 \%$.

\section{IV. ВЫВОДЫ}

Проведенный обзор и экспериментальных исследований теплопроводности и вязкости суспензий с наночастицами позволил оценить влияние основных факторов на свойства переноса нанофлюидов. Приведены результаты экспериментального исследования теплопроводности и вязкости модельной системы изопропиловый спирт - наночастицы $\mathrm{Al}_{2} \mathrm{O}_{3}$ при различных концентрациях наночастиц и температурах. Исследование теплопроводности проводилось двумя независимыми методами стационарным и нестационарным методами нагретой нити. Исследовано также влияние добавки наночастиц на вязкость холодильного компрессорного масла и показано, что добавка наночастиц существенно увеличивает вязкость базовой жидкости, а присутствие маловязкого сурфактанта обеспечивает противоположный эффект. Результаты измерений представлены в виде простых моделей.

\section{ЛИТЕРАТУРА}

1. Шимчук, Н. А. Влияние различных факторов на теплопроводность нанофлюидов [Текст] / Н. А. Шимчук, В. 3. Геллер // Восточно-Европейский журнал передовых технологий. - 2014. - Т. 72, №6 (11). - C. 35-40. doi: 10.15587/17294061.2014.31386

2. Preparation and characterization of nanofluids containing alumina particles [Text] : proceedings of the International Symposium on Thermal and Materials Nanoscience and Nanotechnology, 29 May - 3 June, 2011, Antalya, Turkey

3. Wang, X. A Review on Nanofluids - Part I: Theoretical and Numerical Investigations / X. Wang, A. S. Mujumdar // Brazilian Journal of Chemical Engineering. - 2008. - Vol. 25, No. 04, - P. 613-630. doi: $10.1590 / \mathrm{s} 0104-66322008000400002$ 
4. Maxwell, J. C. A Treatise on Electricity and Magnetism. Second edition [Text] / J. C. Maxwell. Clarendon Press, Oxford, UK, 1881.

5. Hamilton, R. L. Thermal conductivity of heterogeneous two component systems / R. L. Hamilton, O. K. Crosser // Ind. Eng. Chem. Fundamen. - 1962. - №1 (3). - P. 182-191. doi: 10.1021/i160003a005

6. Einstein, A. A new determination of molecular dimensions [Text] / A. Einstein // Ann. Phys. - 1906. - №19. - P. 289-306.
7. Batchelor, G. K. Brownian diffusion of particles with hydrodynamic interaction [Text] / G. K. Batchelor // J. Fluid Mech. - 1976. - Vol. 74, Issue 01. - P. 1-29. doi: 10.1017/s0022112076001663 $\begin{array}{lll}\begin{array}{l}\text { 8. Железный, } \\ \text { экспериментальное } \\ \text { теплофизических }\end{array} & \text { свойств } & \begin{array}{l}\text { исследование } \\ \text { нанофлюидов }\end{array}\end{array}$ изопропиловый спирт $/ \mathrm{Al}_{2} \mathrm{O}_{3}$ на линии кипения [Текст] / В. П. Железный, В. 3. Геллер, Н. А. Шимчук, Н. Н. Лукьянов, А. Г. Никулин // Вестник Казанского технологического университета. 2014. - Т. 17, № 21. - С. 97-99.

V.Z. Geller, N.A. Shimchuk, S.N. Gubanov

Odessa National Academy of Food Technologies, 112, Kanatnaya Str., Odessa, 65039, Ukraine

\section{TRANSPORT PROPERTIES OF NANOFLUIDS (EXPERIMENT AND CALCULATION METHODS)}

$A$ review and analysis of experimental studies and methods of calculation of thermal conductivity and viscosity of nanofluids with $\mathrm{Al}_{2} \mathrm{O}_{3}$ particles are presented. Analysis of published data shows that the results are not consistent. The greatest influence on the thermal conductivity and viscosity of nanofluids make a concentration of the nanoparticles. In addition to concentration, a major factors influencing the transport properties are the following: a method of producing nanofluids and the methods of their preparation for the research; the size and shape of initial nanoparticles; the ability of the nanoparticles to form clusters with different size; temperature and pressure; surface-active substances (surfactants); type and properties of the base fluids; the methodology of the experiment and its adaptation to the specifics of nanofluids. The results of experimental research of thermal conductivity and viscosity for the model system isopropyl alcohol $-\mathrm{Al}_{2} \mathrm{O}_{3}$ nanoparticles at various nanoparticle concentrations and temperatures are given. Study of thermal conductivity was carried out by two independent methods - stady-state and transient hot wire methods. The viscosity was measured with the capillary viscometers. The influence of nanoparticles additives on the viscosity of refrigeration compressor oil is also studied. The results of the measurements are presented in the form of simple models.

Keywords: Thermal Conductivity - Viscosity - Nanofluid - Isopropyl alcohol $-\mathrm{Al}_{2} \mathrm{O}_{3}-$ Compressor oil

\section{REFERENCES}

1. SHIMCHUK, N.A., GELLER, V.Z. 2014. Influence of various factors on the thermal conductivity of nanofluids. Eastern-European Journal of Enterprise Technologies, Vol.72, № 6 (11), pp. 35-40. doi: 10.15587/1729-4061.2014.31386

2. TAVMAN, I., TURGUT, A., CHIRTOC, M., et al., 2011. Preparation and characterization of nanofluids containing alumina particles. Proceedings of the International Symposium on Thermal and Materials Nanoscience and Nanotechnology, Antalya, Turkey

3. WANG, X., MUJUMDAR A.S., 2008. A Review on Nanofluids - Part I: Theoretical and Numerical Investigations. Brazilian Journal of Chemical Engineering, 25(4), pp. 613-630. doi: 10.1590/s0104-66322008000400002

4. MAXWELL, J.C., 1881. A Treatise on Electricity and Magnetism. Second edition. Clarendon Press, Oxford, UK.

5. HAMILTON, R.L., CROSSER, O.K., 1962. Thermal conductivity of heterogeneous two component systems. Ind. Eng. Chem. Fundamen, 1(3), pp. 182-191. doi: 10.1021/1160003a005

6. EINSTEIN, A., 1906. A new determination of molecular dimensions. Ann. Phys, 19, pp. 289-306.

7. BATCHELOR, G.K., 1976. Brownian diffusion of particles with hydrodynamic interaction. $J$.

Fluid Mech., 74(1), pp. 1-29. doi: 10.1017/s0022112076001663 
8. ZHELEZNY, V.P., GELLER, V.Z., SHIMCHUK, N.A., et al., 2014. A comprehensive experimental study of thermal properties of isopropyl alcohol / $\mathrm{Al}_{2} \mathrm{O}_{3}$ nanofluids on the boiling line. Herald of Kazan Technological University, 17(21), pp. 97-99. 